\title{
A compreensão das coordenadas espaciais por crianças de 6 a 8 anos: um estudo exploratório
}

\author{
Ana Coêlho Vieira Selva \\ Jorge Tarcísio da Rocha Falcão \\ Universidade Federal de Pernambuco
}

\begin{abstract}
Resumo
Esta pesquisa analisou o desempenho e estratégias de resolução de problemas de crianças em tarefas que envolviam o uso de coordenadas espaciais. Participaram do presente estudo 36 crianças, igualmente distribuídas em subamostras referentes às classes etárias de 6, 7 e 8 anos. As tarefas solicitadas abrangeram dois conjuntos de itens: a) reproduzir a disposição espacial de um ponto numa folha de papel, a partir de situação de partida noutra folha; b) encontrar a intersecção entre dois pontos. As crianças de cada faixa etária foram distribuídas em subgrupos que diferiam quanto às condições de realização das tarefas: papel em branco, papel quadriculado, papel quadriculado com escala. As crianças mais velhas apresentaram desempenhos significativamente melhores do que as mais novas, principalmente na Tarefa 1. Também o papel quadriculado, principalmente quando incluía a escala, favoreceu o desempenho das crianças. Estes dados sugerem a importância de se considerar a influência dos diferentes suportes representacionais em contexto de educação matemática na escola fundamental.
\end{abstract}

Palavras-chave: Coordenadas espaciais, Desenvolvimento infantil, Conceitos matemáticos.

\begin{abstract}
An exploratory study on the comprehension of spatial coordinates among six to eight-year-old children. This research aimed to analyze both performance and problem-solving strategies concerning the use of spatial coordinates by six to eight years-old children. 36 pupils equally distributed by three age-levels (6, 7 and 8 years of age) were presented to two sets of problems: a) reproduction of the placement of a dot in a Cartesian, bi-axial space, trying to reproduce a previous placement in another bi-axial space; b) finding out the crossingpoint of two moving dots in straight and perpendicular trajectories in a bi-axial Cartesian space. Each agelevel was divided into groups differing in terms of available tools: standard and completely blank sheets of A4 paper, square-lined A4 sheets of paper, square-lined sheets of paper with a written numeric-scale aid. Older pupils showed significantly better performance than younger ones, especially in the first set of problems. The use of square-lined sheets of paper (especially when a numeric-scale was provided) showed also to be an important variable in order to explain better performances of certain groups of pupils. These data suggest the importance of taking into account representational aspects in didactic efforts of conceptual development in early mathematics education.
\end{abstract}

Key words: Spatial coordinates, Child development, Mathematical concepts.

$\mathrm{A}$ compreensão de gráficos vem se caracterizando como uma competência social cada vez mais exigida do cidadão-leitor, na medida em que muito freqüentemente informações veiculadas pela mídia se fazem acompanhar de resumos, quadros e anexos neste formato (Meira, 1997; Monteiro, 1998; Monteiro, Selva \& Ferreira, 2000). Em 1997, o próprio MEC reconheceu a importância de se estimular o desenvolvimento da compreensão de gráficos e tabelas no âmbito da formação escolar, incluindo, a partir dos parâmetros curriculares nacionais para a matemática, o estudo de trata- mento de informações no currículo escolar desde as séries iniciais do ensino fundamental (Brasil, 1997; 1998).

Um aspecto fundamental para a compreensão de gráficos refere-se ao conhecimento sobre coordenadas espaciais em um plano ortogonal ou cartesiano (Wainer, 1992). Se a criança não for capaz de entender que cada ponto em um gráfico representa uma intersecção de informações oriundas dos eixos horizontal e vertical, ela terá bastante dificuldade em interpretar e construir qualquer gráfico. Entretanto, surpreendentemente, não há muitos estudos sobre como as cri- 
anças usam duas informações coordenadas para encontrar uma posição no espaço, nem sobre como emerge tal competência, em termos de aprendizagem-desenvolvimento (para uma resenha abrangente acerca de tais estudos, ver notadamente Shaughnessy, Garfield \& Greer, 1996)

Piaget, Inhelder e Szeminska (1960) constataram, a partir de uma série de situações-problema incluindo a reprodução da localização de um ponto em um espaço bi-dimensional em uma folha de papel a partir da localização deste ponto em outra folha de papel semelhante, que crianças somente a partir de aproximadamente 8 anos é que utilizavam um sistema de coordenadas para localizar um ponto no espaço. Sommerville e Bryant (1985) sugeriram que as dificuldades apresentadas pelas crianças no estudo de Piaget e colaboradores estavam relacionadas ao fato de que, nas tarefas propostas por esta equipe de pesquisadores, as crianças precisavam criar suas próprias coordenadas. Assim, talvez fossem obtidos resultados diferentes se as atividades já fornecessem as coordenadas às crianças.

Em uma série de estudos propostos com esse objetivo, Sommerville e Bryant (1985) analisaram o desempenho de crianças entre 4 anos e meio e 6 anos e meio em tarefas em que, dados eixos perpendiculares com coordenadas marcadas nos mesmos, estas crianças tinham que decidir entre vários pontos qual correspondia às coordenadas indicadas. As crianças mais velhas também resolveram problemas mais complexos em que um dos marcadores da coordenada estava a 45 graus do eixo e o outro marcador era perpendicular. Este tipo de problema mostrou-se mais difícil do que os problemas com ambos marcadores perpendiculares aos eixos. Entretanto, de modo geral, crianças de 5 anos e meio e 6 anos e meio saíramse muito bem, mesmo nos problemas mais complexos, o que parece sugerir que bem antes de 8 anos as crianças já podem utilizar coordenadas espaciais. A Figura 1 apresenta uma ilustração dos eixos perpendiculares utilizados nas tarefas.

Um outro estudo que procurou investigar a compreensão de coordenadas espaciais foi realizado por Magina, Campos, Bryant e Nunes (1999). Estes pesquisadores solicitaram a crianças de 7 e 9 anos que resolvessem uma série de tarefas que envolviam a cópia de algumas figuras geométricas regulares (paralelogramo, retângulo e triângulo) e, também, de um ponto, utilizando papel branco ou quadriculado. Os modelos estavam desenhados em uma folha de papel que ficava à frente e à esquerda da criança e a instrução solicitava que a mesma desenhasse uma figura igualzinha, no mesmo lugar, posição e tamanho em outra folha de papel semelhante à que servia de modelo. As crianças tinham direito a duas tentativas. Após a primeira tentativa, elas podiam colocar o papel que haviam desenhado sobre o modelo para avaliar a sua produção. De modo geral, considerando a localização das figuras copiadas, os resultados mostraram que as crianças apresentaram grandes dificuldades em copiar as localizações exatas das figuras, mesmo na cópia do ponto. Outro dado interessante é que na cópia do ponto, o papel quadriculado favoreceu o uso de coordenadas, desde que os melhores resultados foram observados entre as crianças de ambas as idades que o utilizaram em relação às que usaram o papel branco. Em relação à copia das outras figuras geométricas, os índices de acerto foram bastante baixos. Na análise do posicionamento dos vértices das outras figuras, o escore mais freqüente foi zero, mesmo para o grupo que usou o papel quadriculado, sugerindo que as crianças não utilizaram coordenadas externas ao posicionar as figuras geométricas que estavam sendo copiadas. Entre as figuras geométricas cujas cópias foram solicitadas, os melhores resultados foram obtidos na cópia do retângulo, tanto no que se refere à análise do comprimento dos lados, como em relação à representação do ângulo interno. Observaram ainda um forte efeito do paralelismo na construção do paralelogramo e mais marcadamente do retângulo, ou seja, ao copiarem tais figuras, as crianças mantinham os lados opostos como paralelos.

Os resultados obtidos por Magina e colaboradores são bastante interessantes e trazem um novo elemento para a discussão no que se refere ao uso de coordenadas, qual seja a influência do papel quadriculado como referência, observada basicamente na cópia do ponto. Se o papel quadriculado parece ter favorecido o uso de coordenadas na cópia do ponto, por que tal efeito não foi também observado na cópia das outras figuras? Para uma análise mais detalhada seria interessante conhecermos as estratégias a que tais crianças recorreram para a realização das atividades propostas. Entretanto, estes dados sugerem que vale a pena serem realizadas maio-
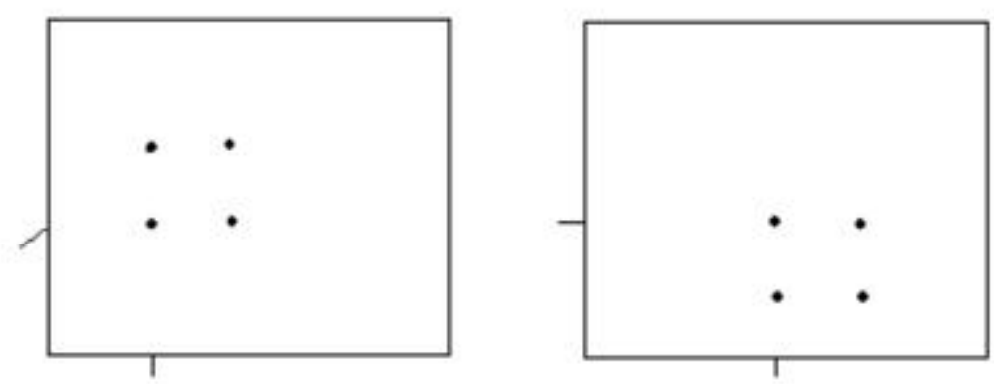

Figura 1

Ilustração dos dois tipos básicos de situações propostas por Sommerville e Bryant (1985), com variação da posição dos marcadores sobre os eixos cartesianos. 
res investigações no que se refere à função do papel quadriculado como um suporte simbólico para o trabalho com coordenadas.

A presente pesquisa procurou analisar o uso de coordenadas em crianças de 6 a 8 anos, a partir de duas tarefas: tarefa de reprodução da localização de um ponto em um espaço bi-dimensional e tarefa de encontro da intersecção entre dois pontos. As crianças de cada idade foram distribuídas em três grupos que diferiam quanto às condições de realização das tarefas: papel em branco, papel quadriculado, papel quadriculado com escala. Neste sentido, este estudo difere dos anteriores na medida em que pretendeu analisar não apenas o desempenho das crianças, mas investigar a influência de diferentes suportes (papel branco, quadriculado e quadriculado com escala) para a realização das tarefas bem como descrever as estratégias utilizadas pelas crianças. Tal objetivo geral é explicitado na seção seguinte.

\section{Método}

\section{Sujeitos}

Foram entrevistadas 36 crianças de uma escola da rede pública da cidade do Recife, sendo 12 crianças de 6 anos (idade média $=6$ anos e 3 meses), 12 de 7 anos (idade média = 7 anos e 4 meses) e 12 de 8 anos (idade média $=8$ anos e 3 meses).

\section{Tarefas}

Cada criança resolveu duas tarefas, sendo a ordem de apresentação controlada. Assim, metade das crianças em cada subgrupo por faixa etária resolveu primeiro a Tarefa 1 e depois a Tarefa 2, enquanto a outra metade fez o inverso.

A Tarefa 1 , referente à reprodução da localização de um ponto em um espaço bi-dimensional, consistia em a partir do desenho de um ponto em dois eixos retangulares, dado um outro desenho de eixos igual ao modelo, desenhar na mesma posição um outro ponto. A Tarefa 2, por sua vez, consistia em encontrar a intersecção entre dois pontos, solicitando-se à criança que dado um par de coordenadas marcadas em dois eixos perpendiculares, indicasse o ponto de intersecção de tais pontos.

Na Tarefa 1, a figura que servia como modelo estava desenhada em um gráfico que se encontrava à esquerda e acima da figura onde a criança deveria copiar o ponto. O par ordenado utilizado em ambas tarefas variou em relação à distância dos eixos: em cada tarefa metade dos pares ordenados eram constituídos do valor de $x$ mais próximo ao eixo, enquanto na outra metade, o valor de $y$ era mais próximo ao eixo.

Com o objetivo de deixar às crianças mais à vontade com as tarefas, foram criadas instruções que faziam alusão a duas formiguinhas muito amigas, de tal forma que uma adorava imitar o que a outra fazia. Assim, a instrução da Tarefa 1 (reprodução da localização de um ponto em um espaço bidimensional) foi a seguinte:

Você está vendo esse ponto? Foi um buraquinho que uma for-
miguinha fez nesta parede. A amiguinha dessa formiguinha, que
adorava imitar tudo que a amiga fazia, encontrou uma parede
igualzinha, do mesmo tamanho que a outra parede. Ela agora
quer fazer um buraquinho no mesmo lugar que a sua amiga fez.
Mas tem que ser no mesmo lugar, exatamente o mesmo lugar.

$\mathrm{Na}$ Tarefa 2 (encontrar o ponto de intersecção entre dois pontos indicados em eixos cartesianos), foi solicitado à criança indicar o local onde as duas formiguinhas iriam se encontrar, saindo cada uma de um eixo e caminhando sempre em linha reta e para frente. Assim, as crianças recebiam uma folha com o desenho de um eixo de coordenadas que tinha, uma formiguinha desenhada em um determinado ponto de cada eixo. A instrução foi a seguinte:

Está vendo esses dois bichinhos, são duas formiguinhas muito amigas, que adoram se encontrar para conversar. Só que tem uma coisa diferente, essas duas formiguinhas só andam para frente e em linha reta. Elas não fazem curvas, não andam para os lados e nem para trás, só em frente e reto. Está vendo cada uma delas? Onde elas vão se encontrar?

Para melhor visualização das tarefas, podemos observar a Figura 2.

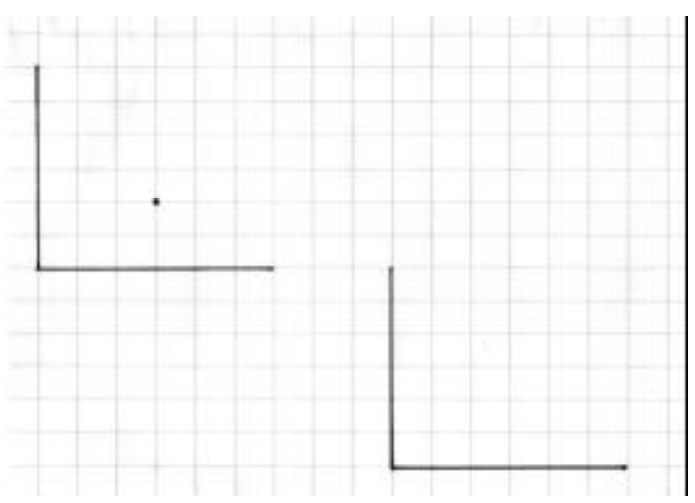

Figura 2

Ilustração das tarefas no grupo com papel quadriculado: Tarefa de localização do ponto de encontro entre os caminhos de duas formiguinhas que só andam para frente e em linha reta (esquerda) e tarefa de cópia da localização de um ponto em outro espaço bi-dimensional semelhante (direita). 
Tabela 1

Constituição dos subgrupos em cada faixa etária, a partir das condições de realização das tarefas propostas

\begin{tabular}{ll}
\hline Subgrupos & Condição de realização das tarefas \\
\hline 1 & Eixos perpendiculares desenhados em papel em branco; \\
2 & Eixos perpendiculares desenhados em papel quadriculado; \\
3 & Eixos perpendiculares desenhados em papel quadriculado, constando \\
& também em cada eixo, uma escala métrica.
\end{tabular}

\section{Procedimento}

As crianças de cada subgrupo por idade foram por sua vez distribuídas em três subgrupos grupos de mesmo efetivo que diferiam em relação às condições para realização das tarefas, conforme resumido na Tabela 1.

Em todos os subgrupos, duas réguas, uma de $30 \mathrm{~cm}$ e outra de $15 \mathrm{~cm}$, foram colocadas ao lado das crianças, com a instrução de que se quisessem poderiam utilizá-las.

Para realização das entrevistas, as crianças foram chamadas a resolver uma atividade com uma "tia" (professora) diferente (a experimentadora). Todas as entrevistas foram gravadas e transcritas literalmente. Numa mesma entrevista as crianças resolviam as Tarefas 1 e 2, na ordem definida, como explicado acima.

\section{Resultados}

Os dados foram analisados quantitativa e qualitativamente. Em relação à análise quantitativa, foi realizada uma análise de variância (ANOVA) com medidas repetidas, tendo como variáveis entre sujeitos a idade (6, 7 e 8 anos), os subgrupos de condição de realização da tarefa (papel em branco, quadriculado e quadriculado com escala) e a ordem de apresentação das tarefas (Tarefa $1 \rightarrow$ Tarefa 2 , Tarefa $2 \rightarrow$ Tarefa 1); como variável intra-sujeitos, a variável tipo de tarefa (ativa ou passiva); a variável dependente considerada foi o desempenho das crianças na tarefa proposta.

Em relação à variável idade, observamos, como seria de se esperar, melhores desempenhos com o aumento da idade. Assim, crianças de 6 anos apresentaram um percentual de acerto nas tarefas de $29,16 \%$, crianças de 7 anos obtiveram
$66,66 \%$ de acerto e, crianças de 8 anos, 79,16\% de acerto. Estas diferenças mostraram-se significativas $(F(18,2)=7,31$, $\mathrm{p}<0,005)$.

Considerando o grupo do qual a criança fazia parte (papel branco, quadriculado e quadriculado com escala) observamos que crianças no grupo papel branco obtiveram um percentual de acerto de $33,33 \%$, no grupo de papel quadriculado, $62,5 \%$ e no grupo papel quadriculado com escala, $79,16 \%$. Estes resultados foram significativos $(F(18,2)=5,81$, $\mathrm{p}<0,011$, sugerindo que o tipo de suporte simbólico disponível para a criança pode interferir significativamente no seu desempenho.

Analisando o desempenho das crianças nas tarefas apresentadas, observamos que a Tarefa 2 foi significativamente mais fácil $(72,22 \%$ de acertos) do que a Tarefa $1(44,44 \%$ de acertos) $(\mathrm{F}(18,1)=12,50, \mathrm{p}<0,002)$. Considerando a interação entre as variáveis grupo e tarefa, verificamos desempenhos semelhantes entre os grupos quando consideramos a Tarefa 2, diferentemente do que ocorre na Tarefa 1, em que há uma melhora de desempenho das crianças em função do grupo. Assim, a interação entre estas variáveis mostrou-se significativa $(F(18,2)=7,63, p<0,004)$. Estes dados podem ser visualizados na Figura 3.

Finalmente, a variável ordem de apresentação das tarefas bem como todas as interações entre variáveis não citadas não apresentaram resultados significativos.

Diante de tais resultados, conduzimos uma análise qualitativa para conhecer melhor como foi o desempenho das crianças.

Em relação à tarefa de reprodução da localização de um ponto em um espaço bi-dimensional, Tarefa 1 , encontramos quatro estratégias:

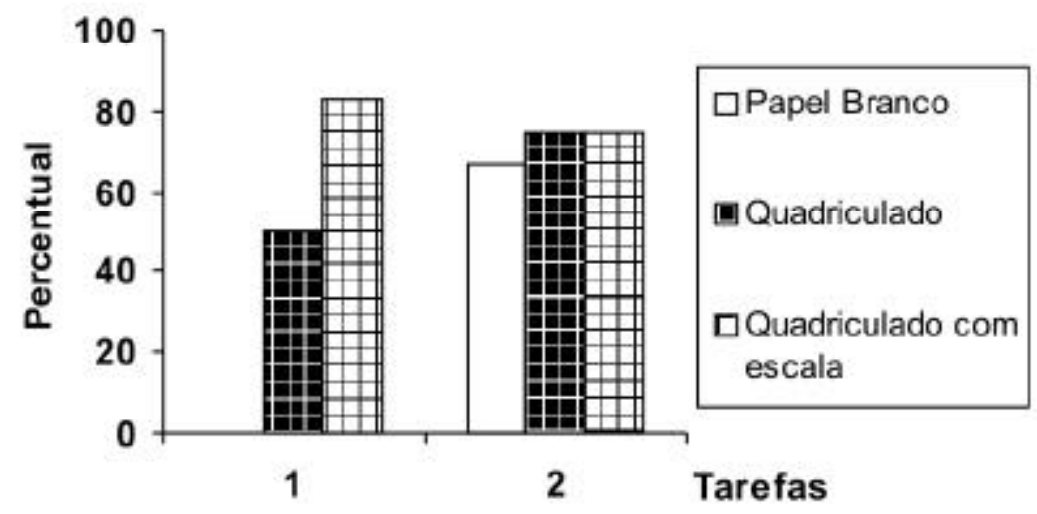

Figura 3

Percentuais de acerto nas tarefas em função do tipo de suporte fornecido 
1. Estratégia perceptual - a criança baseia a cópia do ponto apenas em sua percepção visual. Exemplo: "tô vendo. É aqui", "tá no mesmo lugar que esse, eu olhei e fiz" etc.

2. Considera apenas um dos eixos - a criança conta os quadrados ou linhas no papel quadriculado em relação apenas a um dos eixos, sem considerar o outro. No caso do papel branco, as crianças colocavam o dedinho de um dos eixos até o ponto para medir. Entretanto, esta medição não era feita criteriosamente, nem com uso da régua, era realizada perceptualmente. Exemplo: (no papel quadriculado) "estava contando os buracos (quadrados). Tem um, dois, três (entre o eixo $y$ e o ponto desenhado no modelo). Então é aqui (conta três quadrados a partir do eixo $y$ e faz o ponto)".

Ex2. (no papel em branco) "tá no mesmo lugar. Vou colocar o dedinho (coloca um dedinho do eixo y até o ponto a ser desenhado). É nesta listra (do dedo). Vou botar nesse outro (repete procedimento anterior no outro desenho e marca o ponto) É aqui".

3. Considera os dois eixos por etapas - a criança conta os quadrados ou linhas de um dos eixos até o ponto e transfere esta contagem para a figura a ser copiada. Em seguida, conta também os quadrados ou linhas do outro eixo até o ponto na figura que serve de modelo, de modo a adequar o local do ponto na outra figura. Exemplo: "C: Aqui tem uma linha (entre o eixo x e o ponto). É aqui. E: Está no mesmo lugar que o outro? C: Peraí, um, dois, três (conta 3 quadrados do eixo y para o ponto na figura que serve de modelo). Não é aqui não (onde havia marcado o ponto). É aqui. Contei três quadradinhos aqui e depois fiz o desenho".

4. Uso de coordenadas - a criança considera as coordenadas do ponto na figura que serve como modelo e desenha o ponto de acordo com essas coordenadas. Exemplo: "É aqui. Tem uma linha aqui (distância do eixo x) e três quadrados daqui (distância do eixo y)".
Os percentuais de uso das estratégias nesta tarefa em função da idade e do subgrupo podem ser observados na Tabela 2.

Podemos observar, inicialmente, que o grupo que recebeu o papel em branco apresenta, em todas as idades, os maiores percentuais da estratégia baseada apenas na percepção. Neste sentido, devemos notar que a régua não foi utilizada por nenhuma criança para realizar medições, nem mesmo pelas crianças deste grupo com papel em branco que não contavam com o apoio dos quadradinhos. $\mathrm{O}$ uso desta estratégia perceptual parece, então, contribuir decisivamente para $\mathrm{o}$ alto percentual de erros deste grupo, nesta tarefa, mencionado na análise quantitativa.

Outro dado interessante relativo à estratégia perceptual é que o seu uso parece decrescer com a idade, ao contrário das estratégias que consideram os dois eixos de coordenadas (estratégias 3 e 4), que tem seus usos acrescidos com a idade. A referida relação entre estas estratégias e a variável idade (sem considerar o tipo de tarefa) mostrou-se significativa $\left(X^{2}=6,78 \mathrm{gl}=20,05>\mathrm{p}>0,02\right)^{1}$.

A Tabela 2 apresenta ainda dados que mostram um maior uso das estratégias de uso das coordenadas pelas crianças dos grupos com papel quadriculado, em todas as idades. Considerando as crianças de 6 anos, podemos observar que foram ainda mais favorecidas quando o papel quadriculado incluía uma escala numérica.

Em relação à Tarefa 2, marcar o encontro de duas coordenadas, três tipos de estratégias foram observadas:

1. Alternar o ponto: a criança alternava o lugar que achava que seria aquele onde se daria o encontro das formiguinhas: ora o ponto de encontro estava localizado em um ponto que apenas a formiguinha que vinha do eixo $\mathrm{x}$ passaria, ora $\mathrm{o}$ ponto encontrava-se apenas no caminho da formiguinha que vinha do eixo y. A criança ia alternando pontos em função dos questionamentos do entrevistador, conforme ilustrado

Tabela 2

Distribuição percentual de uso das estratégias na Tarefa 1 em função da idade e do subgrupo por condição de realização da tarefa.

\begin{tabular}{llllll|}
\hline \multirow{5}{*}{ Idade } & & \multicolumn{4}{c|}{ Estratégias } \\
\cline { 3 - 6 } & Subgrupos & $\begin{array}{l}\text { Estratégia 1- } \\
\text { Perceptual }\end{array}$ & $\begin{array}{l}\text { Estratégia 2- } \\
\text { Considera um } \\
\text { dos eixos }\end{array}$ & $\begin{array}{l}\text { Estratégia 3- } \\
\text { Considera os } \\
\text { dois eixos por } \\
\text { etapas }\end{array}$ & $\begin{array}{l}\text { Estratégia 4- } \\
\text { Uso de coorde- } \\
\text { nadas }\end{array}$ \\
\hline 6 anos & Papel branco & 100 & 0 & 0 & 0 \\
& Quadriculado & 75 & 25 & 0 & 0 \\
7 anos & Quad. c/ escala & 50 & 0 & 0 & 50 \\
& Papel branco & 75 & 25 & 0 & 0 \\
& Quadriculado & 50 & 0 & 50 & 0 \\
& Quad. c/ escala & 0 & 0 & 25 & 75 \\
& Papel branco & 75 & 25 & 0 & 0 \\
& Quadriculado & 0 & 0 & 50 & 50 \\
& Quad. c/ escala & 0 & 0 & 50 & 50 \\
\hline
\end{tabular}


pelo fragmento de protocolo abaixo ( $\mathbf{C}$ indicando falas da criança, $\mathbf{E}$ indicando intervenções da experimentadora):

C: É aqui o encontro (ponto que fazia parte da rota apenas da formiguinha $\mathrm{x}$ ).

E: E esta outra formiguinha, ela passa por aí?

C: Não. Então é aqui (ponto da rota da formiguinha y).

$\mathrm{E}$ : E agora, esta formiguinha (do eixo x) passa aí?

C: Não.

E: Então, onde as formiguinhas se encontram?

$\mathrm{C}$ : Aqui (ponto da rota apenas da formiguinha $\mathrm{x}$ ).

2. Fazer os caminhos para marcar o cruzamento: a criança traçava o caminho de uma das formiguinha, superando a indicação da outra coordenada. Depois fazia o mesmo em relação à outra formiguinha. Só depois de fazer ambos os caminhos, a criança marcava o ponto de encontro.

3. Marcar o ponto ou traçar caminho de um eixo até o ponto: a criança marcava apenas o ponto de encontro entre as duas formiguinhas ou fazia o caminho de uma delas apenas até o ponto indicado pelo outro eixo de coordenada.

A Tabela 3 apresenta os resultados da análise das estratégias na Tarefa 2 em função da idade e do grupo. Podemos observar que também nesta tarefa, os grupos que tiveram papel quadriculado apresentaram estratégias mais avançadas, ou seja, consideraram os dois eixos de coordenadas para marcar o ponto de encontro das formiguinhas, do que as crianças do grupo com papel branco. Entretanto, ao contrário da Tarefa 1, o desenho da escala não pareceu influenciar no uso das estratégias por parte das crianças.

Um outro dado interessante refere-se ao decréscimo do uso da estratégia de alternar os eixos com a idade, o que sugere um aumento na consideração do eixo de coordenadas para a localização de um ponto.

Finalmente, a distinção entre as estratégias de fazer os caminhos para depois marcar o ponto e de fazer o ponto já considerando os eixos de coordenadas parece indicar graus de complexidade diferentes em termos da consideração dos eixos de coordenadas. Ao fazer os caminhos, a criança parece sentir necessidade, inicialmente, de focalizar cada coordenada independentemente da outra, enquanto que ao apenas marcar o ponto ou mesmo, seguir uma das coordenadas até o ponto indicado pela outra (mas sem desenhar o outro caminho) sugere uma consideração simultânea dos dois eixos de coordenadas.

\section{Conclusões}

Inicialmente devemos notar que enquanto crianças de 7 e 8 anos apresentaram um bom desempenho nas tarefas solicitadas, crianças de 6 anos apresentaram bastante dificuldades, principalmente no que se refere à tarefa de cópia do ponto. De modo geral, esta tarefa foi mais difícil para as crianças de todas as idades, principalmente nos grupos com papel branco. É interessante salientar que mesmo crianças que resolveram primeiro a tarefa de encontrar a intersecção de dois pontos, encontraram dificuldades em usar coordenadas para resolver a tarefa de cópia.

Em relação à tarefa de encontrar a intersecção entre dois pontos, os resultados obtidos neste estudo contrastam com os obtidos por Sommerville e Bryant (1985). Enquanto estes pesquisadores observaram excelentes desempenhos de crianças a partir de 5 anos e meio quando se ofereciam as coordenadas, encontramos muitas dificuldades ainda entre as crianças de 6 anos. Uma possível explicação consiste no tipo de tarefa apresentado. Enquanto no estudo desses pesquisadores eram oferecidos pontos para que a criança indicasse qual representava a intersecção entre as coordenadas dadas, em nosso estudo, a criança não tinha tais pontos desenhados no eixo retangular. Parece interessante, então, que novas pesquisas na área explorem estas diferenças.

Um outro aspecto que deve ser enfatizado consiste no uso quase ausente da régua por todas as crianças como ferramenta auxiliar nas tarefas propostas. Esperávamos que ao

Tabela 3

Distribuição percentual de uso das estratégias na Tarefa 2 em função da idade e do subgrupo por condição de realização da tarefa.

\begin{tabular}{lllll|}
\hline \multirow{2}{*}{ Idades } & Subgrupos & Alterna & $\begin{array}{l}\text { Faz os caminhos } \\
\text { e marca o } \\
\text { cruzamento }\end{array}$ & $\begin{array}{l}\text { Marca o ponto ou } \\
\text { traça caminho de } \\
\text { um eixo até o ponto }\end{array}$ \\
\cline { 3 - 5 } 6 anos & Papel branco & 50 & 25 & 25 \\
& Quadriculado & 50 & 0 & 50 \\
& Quad. c/ escala & 75 & 0 & 25 \\
7 anos & Papel branco & 25 & 50 & 25 \\
& Quadriculado & 25 & 0 & 75 \\
& Quad. c/ escala & 0 & 0 & 100 \\
& Papel branco & 25 & 50 & 25 \\
& Quadriculado & 0 & 25 & 75 \\
& Quad. c/ escala & 0 & 25 & 75 \\
\hline \multirow{7}{*}{ anos } & & & &
\end{tabular}


menos as crianças mais velhas que estavam no grupo com papel em branco utilizassem a régua, na tarefa de reprodução da localização de um ponto em um espaço bi-dimensional, para medirem as distâncias do ponto aos eixos. Entretanto, nas poucas vezes em que a régua foi utilizada, o objetivo de seu uso foi, simplesmente, a construção de uma linha reta, não sendo usada como instrumento de medida. Ainda em relação ao uso da régua, é interessante notar que as crianças de 7 e 8 anos do grupo com papel branco que tentaram fazer a medição de pelo menos um dos eixos de coordenadas na tarefa de reprodução da localização de um ponto em um espaço bi-dimensional, preferiram utilizar o dedo como instrumento de medida, o que parece também indicar uma certa falta de familiaridade das crianças com a régua enquanto instrumento de medida.

Também merece ser comentado o efeito do papel quadriculado na realização das tarefas, em todas as idades. A presença do papel quadriculado (com ou sem escala) parece ter favorecido o uso das coordenadas para a execução de ambas as tarefas. Na tarefa de reprodução da localização de um ponto em um espaço bi-dimensional, a inclusão da escala (grupo 3) também parece ter influenciado as crianças de 6 e 7 anos, possibilitando o uso de estratégias mais avançadas de uso das coordenadas. Estes dados parecem indicar a necessidade de se considerar os suportes simbólicos na compreensão do conhecimento lógico-matemático das crianças, ressaltando a importância de se proporcionar situações realmente estimuladoras no processo de ensino-aprendizagem da matemática.

Finalmente, devemos considerar o fato de não termos encontrado diferenças de desempenho em função da ordem de apresentação das tarefas, ainda que a tarefa de encontrar a intersecção entre dois pontos tenha sido significativamente mais fácil do que a tarefa de reprodução da localização de um ponto em um espaço bi-dimensional. Estes dados sugerem a importância de se realizar na escola um trabalho simultâneo entre construção e interpretação, desde que tais ativi- dades ainda que relacionadas, parecem guardar uma certa independência entre si. Ainda, do ponto de vista educacional, este estudo mostra que a compreensão de coordenadas merece uma maior atenção por parte dos professores, principalmente quando sabemos que os parâmetros curriculares sugerem o trabalho na área de tratamento de informações, incluindo a representação gráfica, a partir da primeira série do ensino fundamental. Parece, então, interessante a realização de novos estudos que ampliem a discussão dos resultados aqui obtidos a partir de situações mais contextualizadas para as crianças do que as utilizadas na presente pesquisa.

\section{Referências}

Brasil. (1997). Parâmatros curriculares nacionais: matemática. Brasília: MEC/ SEF.

Brasil. (1998). Referência curricular nacional para a educação infantil. Brasília: $\mathrm{MEC} / \mathrm{SEF}$

Magina, S., Campos, T., Bryant, P., \& Nunes, T. (1999). A concepção de espaço da criança: um estudo diagnóstico. Manuscrito não-publicado.

Meira, L. (1997). Gráficos de quantidades na vida diária e na mídia impressa. Trabalho apresentado na II Semana de Estudos em Psicologia da Educação Matemática, Departamento de Psicologia, UFPE, Recife, PE.

Monteiro, C. E. F. (1998). Interpretação de gráficos sobre economia veiculados pela mídia impressa. Dissertação de mestrado não-publicada. Universidade Federal de Pernambuco, Recife.

Monteiro, C. E. F., Selva, A. C. V., \& Ferreira, J. C. S. (2000). Tratamento de informações: investigando o processo de interpretação de gráficos [Resumo]. In Sociedade Brasileira para Progresso da Ciência (Org.), Anais da $52^{\mathrm{a}}$ Reunião Anual da Sociedade Brasileira para o Progresso da Ciência (CDROM). Brasília: Autor.

Piaget, J., Inhelder, B., \& Szeminska, A. (1960). The child's conception of geometry. New York: Norton.

Sommerville, S., \& Bryant, P. (1985). Young children's use of spatial coordinates. Child Development, 56, 604-613.

Shaughnessy, J. M., Garfield, J., \& Greer, B. (1996). Data handling. In A. J. Bishop, M. A. Clements, C. Keitel, J. Kilpatrick, J. \& C. Laborde (Orgs.). International handbook of mathematics education (Parte 1, pp. 205-238). London: Kluwer.

Wainer, H. (1992). Understanding graphs and tables. Educational Researcher, $21,14-23$.

Nota

1 Qui-quadrado realizado a partir das frequências obtidas, considerando-se apenas as variáveis idade (6,7 e 8 anos)

e tipo de estratégia (perceptual e as que consideram os dois eixos de coordenadas).

Ana Coêlho Vieira Selva, mestre e doutoranda em Psicologia em Psicologia pela Universidade Federal de Pernambuco, é Professora Assistente do Departamento de Psicologia e Orientação Vocacional da Universidade Federal de Pernambuco.

Jorge Tarcísio da Rocha Falcão, doutor em Psicologia da Aprendizagem pela Universite de Paris V (Rene Descartes), França, é Professor Adjunto do Departamento de Psicologia da Universidade Federal de Pernambuco.

Endereço para correspondência: Pós-Graduação em Psicologia - UFPE, Av. Acadêmico Hélio Ramos, s/n CFCH $-8^{\circ}$ andar, Cidade Universitária, 56700-901, Recife, PE. Fones: (81)3271.8272/3271.1843 Fax: (81)327.1843. E-mail: [ACVS] anaselva@elogica.com.br; [JTRF] jtrf@npd.ufpe.br. 